\title{
Cost Effectiveness of Different Strategies for Detecting Cirrhosis in Patients With Non-alcoholic Fatty Liver Disease Based on United States Health Care System
}

\section{Short title: Non-invasive diagnosis of cirrhosis in NAFLD}

${ }^{1}$ Eduardo Vilar-Gomez ${ }^{*},{ }^{2}$ Zhouyang Lou*, ${ }^{3}$ Nan Kong**, ${ }^{1}$ Raj Vuppalanchi, ${ }^{1,4,5}$ Thomas F Imperiale**, ${ }^{1}$ Naga Chalasani**

${ }^{1}$ Department of Medicine, Indiana University School of Medicine, Indianapolis, IN

${ }^{2}$ School of Industrial Engineering, Purdue University, West Lafayette, IN

${ }^{3}$ Weldon School of Biomedical Engineering, Purdue University, West Lafayette, IN

${ }^{4}$ Center for Innovation, Health Services Research and Development, Roudebush VA Medical Center

$$
\begin{gathered}
{ }^{5} \text { Regenstrief Institute, Inc. } \\
{ }^{*} \text { Contributed Equally } \\
* * \text { Co-corresponding authors }
\end{gathered}
$$

Short title: Non-invasive strategies for cirrhosis in NAFLD

Funding: No external funding supported this study.

Corresponding Authors: Naga Chalasani (nchalasa@iu.edu), Thomas F. Imperiale (timperia@iu.edu), or Nan Kong (nkong@purdue.edu)

This is the author's manuscript of the article published in final edited form as:

Vilar-Gomez, E., Lou, Z., Kong, N., Vuppalanchi, R., Imperiale, T. F., \& Chalasani, N. (2020). Cost Effectiveness of Different Strategies for Detecting Cirrhosis in Patients With Non-alcoholic Fatty Liver Disease Based on United States Health Care System. Clinical Gastroenterology and Hepatology. https://doi.org/10.1016/j.cgh.2020.04.017 


\section{Abbreviations}

NAFLD: Nonalcoholic fatty liver disease; VCTE: Vibration controlled transient elastography; MRE: Magnetic resonance elastography; CLD: Chronic liver disease; NITs: non-invasive tests; LB: Liver biopsy; AST: Aspartate aminotransferase; ALT: Alanine aminotransferase; TP: True positive; FP: False negative; TN: True negative; FN: False negative; EV: Esophageal varices; HCC: Hepatocellular carcinoma; NASH: Nonalcoholic steatohepatitis; OLT: Orthotopic liver transplantation; AASLD: American association for the study of the liver diseases; CC: Correctly classified; AFP: Alpha-fetoprotein; NSBB: Nonselective beta-blockers; GI: Gastrointestinal; CPDP, cost per death prevented.

\section{Author Contributions}

Eduardo Vilar-Gomez: Study design, data collection and analysis, manuscript preparation, final approval

Raj Vuppalanchi: Study design, data collection and analysis, manuscript preparation, final approval

Naga Chalasani: Study design, data collection and analysis, manuscript preparation, final approval Nan Kong: Study design, data collection and analysis, modelling, manuscript preparation, final approval

Zhouyang Lou: Study design, data collection and analysis, modelling, manuscript preparation, final approval

Thomas F. Imperiale: Study design, data collection and analysis, modelling, manuscript preparation, final approval

\section{Authors Emails}

Eduardo Vilar-Gomez evilar@iu.edu

Zhouyang Lou zlou@purdue.edu

Nan Kong: nnkong@purdue.edu

Raj Vuppalanchi: $\quad$ rvuppala@iu.edu

Thomas Imperiale timperia@iu.edu

Naga Chalasani: nchalasa@iu.edu 


\section{ACKNOWLEDGEMENTS}

We thank Ms. Carolina Vivas-Valencia from Purdue Weldon School of Biomedical Engineering for her participation in initial meetings on decision tree construction and conceptualization of the microsimulation. Authors thank Mr. Robert W. Klein from Medical Decision Modeling Inc. for his thoughtful review and suggestions. Authors also thank Drs. Samer Gawrieh and Niharika Samala for their comments regarding study design and results. Authors thank Julianne Nanzer for her editorial assistance.

\section{Conflicts of Interests:}

There are none for this paper. For full disclosure, Dr. Chalasani has ongoing paid consulting activities (or had in preceding 12 months) with NuSirt, Abbvie, Afimmune (DS Biopharma), Allergan (Tobira), Madrigal, Coherus, Siemens, La Jolla, Foresite labs, and Genentech. These consulting activities are generally in the areas of nonalcoholic fatty liver disease and drug hepatotoxicity. Dr. Chalasani receives research grant support from Exact Sciences, Intercept, and Galectin Therapeutics where his institution receives the funding. Over the last decade, Dr. Chalasani has served as a paid consultant to more than 35 pharmaceutical companies and these outside activities have regularly been disclosed to his institutional authorities. 


\begin{abstract}
Background \& Aims: Several strategies are available for detecting cirrhosis in patients with nonalcoholic fatty liver disease (NAFLD), but their cost effectiveness is not clear. We developed a decision model to quantify the accuracy and costs of 9 single or combination strategies, including 3 noninvasive tests (fibrosis-4 [FIB-4], vibration controlled transient elastography [VCTE], and magnetic resonance elastography [MRE]) and liver biopsy, for detection of cirrhosis in patients with NAFLD.
\end{abstract}

Methods: Data on diagnostic accuracy, costs, adverse events, and cirrhosis outcomes over a 5-y period were obtained from publications. The diagnostic accuracy, per-patient cost per correct diagnosis of cirrhosis, and incremental cost-effectiveness ratios (ICER) were calculated for each strategy for base cirrhosis prevalence values of $0.27 \%, 2 \%$, and $4 \%$.

Results: The combination of the FIB-4 and VCTE identified patients with cirrhosis in NAFLD populations with a $0.27 \%, 2 \%$, and $4 \%$ prevalence of cirrhosis with the lowest cost per person (\$401, \$690, and \$1024, respectively) and highest diagnostic accuracy $(89.3 \%, 88.5 \%$, and $87.5 \%$ respectively). The combination of FIB-4 and MRE ranked second in cost per person $(\$ 491, \$ 781$, and $\$ 1114$, respectively) and diagnostic accuracy $(92.4 \%, 91.6 \%, 90.6 \%$, respectively). Compared to the combination of FIB-4 and VCTE (least costly), the ICERs were lower for the combination of FIB-4 and MRE (\$2864, \$2918, and \$2921) than the combination of FIB-4 and liver biopsy (\$4454, $\$ 5156$, and \$5956) at the cirrhosis prevalence values tested. When goal was to avoid liver biopsy, FIB-4+VCTE and FIB-4+MRE had similar diagnostic accuracies, ranging from $87.5 \%$ to $89.3 \%$ and $90.6 \%$ to $92.4 \%$ for cirrhosis diagnosis, although FIB-4+MRE had a slightly higher cost.

Conclusions: In our cost effectiveness analysis based on United States health care system, we found that results from FIB-4, followed by either VCTE, MRE, or liver biopsy, detect cirrhosis in patients with NAFLD with a high level of accuracy and low cost. Compared to FIB-4 + VCTE which was the least costly strategy, FIB-4+MRE had lower ICER than FIB-4+LB.

KEY WORDS: comparison, expense, sensitivity, imaging, biomarker 


\section{What You Need to Know}

Background: Several methods are available for detecting cirrhosis in patients with non-alcoholic fatty liver disease (NAFLD), but little is known about their cost effectiveness.

Findings: In an analysis of data from publications, we found that FIB-4, followed by either VCTE, MRE, or liver biopsy, detects cirrhosis in patients with NAFLD with a high level of accuracy and low cost. However, the combination of FIB-4 and liver biopsy has a higher ICER, compared to the combination of FIB-4 and VCTE or FIB-4 and MRE.

Implications for patient care: The FIB-4, in combination with VCTE, MRE or liver biopsy, identifies patients with NAFLD who have cirrhosis accurately and cost effectively. 


\section{INTRODUCTION}

Nonalcoholic fatty liver disease (NAFLD) is a leading cause of chronic liver disease, hepatocellular carcinoma (HCC), and liver transplantation. ${ }^{1}$ The extent of hepatic fibrosis is the strongest risk factor for long-term complications; patients with advanced fibrosis are at the highest risk of hepatic decompensation, HCC, and cardiovascular disease. ${ }^{2}$ Thus, accurate staging of fibrosis is necessary for proper management of patients with NAFLD, both for estimating prognosis and guiding therapy. While liver biopsy (LB) is the gold standard for staging liver fibrosis and diagnosing cirrhosis, it is invasive, costly, and has technical limitations, including sampling error and variation in histological interpretation, making it impractical as a routine screening or diagnostic tool.

Several non-invasive tests (NITs) are available for staging liver fibrosis, including bloodbased biomarkers (Fib-4) ${ }^{3}$, and modalities that assess liver stiffness such as vibration controlled transient elastography (VCTE) ${ }^{4}$ and magnetic resonance elastography (MRE). ${ }^{5}$ Most NITs are good at excluding advanced fibrosis (negative predictive value >90\%), but sensitivity is lower for detecting advanced fibrosis. ${ }^{6-8}$ Among the modalities assessing liver stiffness, VCTE is the most widely studied and validated method, yielding good intra- and inter-observer variability and low failure rates. ${ }^{4}$ Moreover, its wide availability and easy-to-operate feature make VCTE a potential point-of-care test. Most recently, 2D- and 3D-MRE have emerged as promising noninvasive tests for diagnosing advanced fibrosis in NAFLD patients. MRE and VCTE have high accuracy in identifying advanced fibrosis. ${ }^{8}$ The Fib-4 index is a simple and inexpensive blood-based panel that is useful for excluding advanced fibrosis. ${ }^{3}$ It has been extensively validated in patients with NAFLD. Fib-4 cutoffs $\geq 3.25$ or $\leq 1.30$ have $80 \%$ and $90 \%$ positive and negative predictive values, 
respectively, for detecting or excluding advanced fibrosis among patients CLD, including NAFLD. ${ }^{7}$ Its major limitation is that a high proportion of values fall in the indeterminate range (1.30-3.25). ${ }^{7}$

In clinical practice, a sequential combination of two NITs may improve the detection of patients with advanced fibrosis and reduce the number of patients with indeterminate results; ${ }^{9}, 10$ however, its cost-effectiveness has not been fully explored. Herein, we developed a decision model to quantify the accuracy and costs of various non-invasive strategies for diagnosing cirrhosis in NAFLD patients to understand better which strategies might be considered preferable in clinical practice.

\section{METHODS}

\section{Study population}

Our study population consists of a hypothetical cohort of middle-aged patients with NAFLD seen in three different settings: (a) in a specialty clinic setting where the prevalence of cirrhosis in NAFLD is estimated to be $2 \%^{11}$, (b) in a general population-based setting where the prevalence of cirrhosis is estimated to be $0.27 \%^{12}$ and tertiary referral centers setting where cirrhosis prevalence is reported to be $\approx 4 \%$. $5,7,8$

\section{Model structures}

We constructed a decision model to compare the accuracy and costs associated with liver biopsy and NITs alone and in a sequential combination for the diagnosis of cirrhosis. We considered Fib-4 and liver stiffness measured by VCTE or MRE, and assumed that liver biopsy, as the reference standard, would have the highest sensitivity and specificity for cirrhosis. Test 
accuracy for each strategy was calculated as follows: (true positive $(\mathrm{TP})+$ true negative $(\mathrm{TN})) /($ total population). To evaluate the ensuing clinical events for future costs and outcomes; we attached a microsimulation to each leaf node of the decision tree to emulate subsequent clinical events over a 5-year time horizon. Figures 1A-C summarize the decision tree of the nine diagnostic strategies and the transitions in the microsimulation of patients after receiving a diagnosis of cirrhosis.

In the decision-tree portion of the model, we considered each test individually and in clinically appropriate combinations where either stiffness-based method was added sequentially to Fib-4 or where LB was added sequentially to either a stiffness-based method or Fib-4. For all test strategies, a positive test may suggest cirrhosis. Additionally, failure rates for VCTE $(7.1 \%)^{4,13}$ and MRE $(4 \%)^{14,15}$, and indeterminate results for Fib-4 $(32 \%)^{4}$ were included. For combination strategies, a second test was added if the initial test was either positive or indeterminate for Fib-4, or uninterpretable for VCTE/MRE. The result of the second test determined the final diagnosis of cirrhosis. Finally, a do-nothing approach was included along with all test strategies.

We assumed that all patients with cirrhosis diagnosis underwent periodic guideline-based screening for esophageal varices $(\mathrm{EV})$ and $\mathrm{HCC}^{16,17}$ over a 5-year period. The disease state and mortality status were updated during the 5-year period. As a result, costs of the periodic screening for EVs and HCC were accumulated over the 5-year period. For those who tested positive, the costs include: (1) periodic EV screening [every 2 years if EV is small and at 3-year intervals if EVs are absent $],{ }^{17}$ (2) HCC screening every 6 months using serum $\alpha$-fetoprotein and ultrasound, ${ }^{16}$ (3) primary prophylaxis of EV bleeding with both endoscopic band ligation (EBL) and nonselective beta-blockers (NSBB), ${ }^{17}$ (4) outpatient clinic visit and (5) those derived from liver transplantation or HCC-related liver resection. For those who tested negative, the two screening costs as above were excluded. 


\section{Model Parameters}

We performed a sensitivity analysis by varying the prevalence range from $0.27 \%$ to $4 \%$. The prevalence of $\mathrm{EV}$ among cirrhotics was estimated to be $34.7 \% .^{18}$ We considered the annual incidence of EV and $\mathrm{HCC}$ to be $2.3 \%$ (unpublished data from Indiana University Medical Center) and $2.5 \%$, respectively. ${ }^{2}$ The pooled 3 -year HCC mortality rate is $49.2 \%$ among patients undergoing HCC surveillance vs $72.1 \%$ among those without periodic surveillance. ${ }^{16}$ We assumed a $16.3 \%$ 6month risk of mortality due to variceal bleeding. ${ }^{19}$ We considered a prevalence of $11.5 \%$ of large or high-risk varices among compensated NAFLD cirrhotics. ${ }^{20}$ and assumed that $34 \%$ of small or no varices would progress to large varices at 3 years. ${ }^{21}$ The pooled 2-year risk of upper gastrointestinal bleeding is $17 \%^{22}$ and $31 \%^{23}$ among patients with or without primary prophylaxis including both EBL and/or NSSB. Table 1, Figure 1C and supplemental Tables 1-3 summarize some of the above-mentioned parameters used in the model.

An institutional cost perspective was adopted, expressed in 2017 U.S. dollars. Direct costs for LB, MRE and VCTE were obtained from Medicare reimbursement data (supplemental Table 4).

\section{Analysis}

We simulated the costs for a particular strategy, which included the costs of (1) each diagnostic strategy, (2) HCC and EV screenings, (3) periodic clinic visits, (4) management of LBrelated complications (i.e., bleeding) and (5) liver transplantation or HCC-related liver resection. We also simulated the diagnostic accuracy for a particular strategy. Diagnostic accuracy was defined as the percentage of correct diagnosis, which equals the sum of numbers of people who were correctly identified with or without cirrhosis (excluding the numbers after the confirmation 
test), divided by the total population. We next calculated the cost per correct diagnosis, which was used as a proxy in the ensuing cost-effectiveness analysis. We lastly calculated the incremental cost-effectiveness ratios (ICERs) and cost per death prevented (CPDP). ICERs were defined as the incremental cost for each additional correct diagnosis (see supplemental material): the strategies were sorted by ascending order of cost, and the current least costly strategy was compared with the previous least costly strategy. If the accuracy of the current least costly strategy was lower than the previous one, then the current strategy was considered dominated. CPDP was calculated using the same logic, and we used mortality instead of diagnostic accuracy.

We further plotted per-patient total cost and diagnostic accuracy, and per-patient total cost and mortality for each strategy. Any strategy, such that no other strategies would yield lower cost and higher accuracy (lower mortality) simultaneously, was considered to be a dominant strategy. In the presence of more than one dominant strategy, the dominant strategies formed an efficiency frontier; strategies below and to the right of the frontier were considered to be dominated.

To assess the robustness of model results, we performed sensitivity analyses on cirrhosis prevalence $(0 \%-12 \%)$. In addition, we performed one-way sensitivity analyses on test characteristics and costs with cirrhosis prevalence being fixed at $0.27 \%$.

\section{RESULTS}

\section{Base case.}

Low prevalence of cirrhosis $(0.27 \%)$. Fib-4 alone correctly classifies the lowest percentage of persons (57\%), while Fib-4+LB correctly classified 97.7\%, the highest percentage (Table 2). Figure 2A shows diagnostic accuracy by cost per person for each strategy. The solid line represents the efficiency frontier, which identifies strategies with the lowest cost and highest accuracy, and 
includes: Fib-4+VCTE (89.3\%, \$401), Fib-4+MRE (92.4\%, \$491) and Fib-4+LB (97.7\%, \$729). Relative to Fib-4+VCTE, which is the least costly strategy with $89.3 \%$ accuracy, ICERs for the frontier strategies range from $\$ 2,864$ per additional correct diagnosis for Fib-4+MRE to $\$ 4,454$ for Fib-4+LB (Table 2). Figure 2B and supplemental Table 5 display mortality by cost per person for each strategy. Compared to do-nothing strategy, which yields highest mortality (46 deaths) and lowest cost (\$10), Fib-4+VCTE (41 deaths, \$401), VCTE+LB (39 deaths, \$613), VCTE (38 deaths, \$901) and LB (36 deaths, \$1663) show the best combination of cost versus mortality.

Supplemental Tables 6-14 depict detailed results on diagnostic accuracy for all strategies.

Intermediate prevalence of cirrhosis (2\%). Both the cost per person for each strategy and the cost per correct diagnosis per person are higher for an intermediate prevalence of cirrhosis as compared with low cirrhosis prevalence. Fib-4 alone remains the least accurate strategy (56.5\%). Base results for intermediate cirrhosis prevalence closely parallel results for low prevalence with the same three least costly strategies which are Fib-4+VCTE (88.5\% accuracy, cost of \$690), Fib-4+MRE (91.6\%, \$781), and Fib-4+LB (97\%, \$1,060) (Table 3). VCTE+LB has the same cost than Fib-4+LB, but it yields lower diagnostic accuracy (Table $\mathbf{3}$ and Figure 2C). Figure 2C shows 3 strategies on the efficiency frontier, including the three same frontier strategies for the low prevalence case, with ICERs ranging from $\$ 2,918$ (Fib-4+MRE) to $\$ 5,156$ (Fib-4+LB) per additional correct diagnosis (Table 3). Results based on cost versus mortality analysis (Figure 2D and supplemental Table 15), show the same four strategies on the frontier (Fib-4+VCTE [307, \$690], VCTE+LB [285, \$1,060], VCTE [279, \$1,347] and LB [270, \$2,183]) than those results based on low cirrhosis prevalence.

High prevalence of cirrhosis (4\%). Baseline results for the high prevalence of cirrhosis closely parallel those from low and intermediate prevalence (Table 4, Figure 2E). The same three 
strategies (Fib-4 followed by either VCTE, MRE or LB) comprise the 3 least costly strategies, with accuracies ranging from $87.5 \%$ for Fib-4+VCTE to $96.1 \%$ for Fib-4+LB. As expected due to a higher proportion of cirrhosis, both costs per person and cost per correct diagnosis are higher than in the two previous prevalence scenarios, ranging respectively from $\$ 1,024$ for Fib-4+VCTE to $\$ 1,441$ for Fib-4+LB and from $\$ 1,170$ for Fib-4+VCTE to $\$ 1,500$ for Fib-4+LB. Fib-4 alone remains the least accurate strategy (55.9\%). Similar to findings under conditions of intermediate prevalence, the same three frontier strategies appear on the efficiency frontier, with ICERs ranging from $\$ 2,921$ to $\$ 5,956$ per additional correctly diagnosed case. Cost versus mortality analysis also show the same four diagnostic strategies on the frontier, with Fib-4+VCTE displaying highest mortality (613) followed by VCTE+LB (568), VCTE (559) and LB (540) (Figure 2F and supplemental Table 16).

\section{Sensitivity analyses.}

Prevalence of cirrhosis. When the prevalence of cirrhosis is extended to as high as $12 \%$, cost per correct diagnosis increases for all strategies, as shown in Supplemental Figure 2. The largest cost increases are observed for Fib-4, VCTE, and MRE, with respective increases of $\$ 4,276, \$ 4,030$, and $\$ 3,732$ from the previously considered low prevalence. Cost increases are the lowest for the two NITs of FIB-4+VCTE and FIB-4+MRE, with respective increases of $\$ 2,364$ and $\$ 2,298$ from the previously considered low prevalence.

Test characteristics. Results of one-way sensitivity analyses on each test's sensitivity and specificity are shown in Supplemental Figure 3A-D. Within the ranges of test characteristics, the general pattern of cost per correct diagnosis are comparable: negligible effects of sensitivity on either individual or combination strategies, negligible effects of specificity for combination strategies, and a modest cost reduction for individual tests as specificity increases. 
Test costs. Results of 8 one-way sensitivity analyses on the cost of each test are shown in supplemental Table 17 and in supplemental Figure 4. Note that the baseline values were set at Medicare average price supplemental Table 4, i.e., \$0, \$1,411, \$150.34 and \$544.18 for Fib-4 LB, VCTE and MRE. For the one-way sensitivity analysis on each cost parameter, alternative cost values are based on the national average for facility fees among hospital-based clinics and the $90^{\text {th }}$ percentile for charges submitted by hospital-based clinics; see supplemental Table 17.

Considering sensitivity in the tradeoff of cost vs accuracy with respect to the cost parameters, supplemental Table 18 shows the percentage of times ( 9 in total) each strategy is on efficiency frontier. Fib-4+VCTE, Fib-4+MRE, and Fib-4+LB are on efficiency frontier in $89 \%$ of the scenarios, respectively. In contrast, MRE+LB and VCTE+LB are on the frontier only when the costs of MRE and LB are high, and in $11 \%$ of the scenarios, respectively. The Tornado plot of cost per correct diagnosis shows Fib-4+VCTE to be the least costly strategy with a range of $\$ 397$ to \$590, whereas LB and MRE, each alone, are the two most costly strategies (supplemental Figure 4).

\section{DISCUSSION}

Since cirrhosis is the major determinant of long-term morbidity and mortality in patients with NAFLD, there is a critical need to detect cirrhosis before complications occur, which are associated with a high mortality and increased healthcare utilization. The sequential combination of two NITs or a NIT test plus liver biopsy may detect cirrhosis more accurately, ${ }^{9,}{ }^{10}$ however, whether these approaches are cost-effective is unknown. This study suggests that the use of Fib-4 followed by VCTE is likely the most cost-effective strategy for screening or detecting cirrhosis among 
patients with NAFLD either in the setting of general population, or specialty clinics and tertiary referral centers. Other diagnostic strategies such as Fib-4 followed by either MRE or LB, and VCTE followed by LB are likely cost-effective approaches but might have higher costs than Fib-4 followed by VCTE across all cirrhosis prevalence scenarios.

There is a current knowledge gap regarding the cost-effectiveness of single versus sequential combined screening strategies for cirrhosis in NAFLD. Our findings support previous work suggesting that the use of sequential combination tests is more cost-effective than are single tests. $9,10,24$

Among the 5 combination tests compared in the base case analysis, Fib-4+VCTE ranks first due to the best combination of cost per correct diagnosis and diagnostic accuracy. Fib-4+MRE ranks second, with higher percentage of people correctly classified (ranging from $90.6 \%-92.4 \%$ vs. 87.5\%-89.3\% with Fib-4+VCTE) but higher costs. The combinations of Fib-4+LB or VCTE+LB ranked third and fourth, respectively, across all cirrhosis prevalence settings. Although both combinations yield higher accuracy, they have higher costs per correct diagnosis as compared with either FIB-4+VCTE or Fib-4+MRE. Finally, the combination of MRE+LB ranked fifth among all combination tests.

Overall, using MRE together with either LB or Fib-4 moderately increases overall costs and therefore reduces cost-effectiveness as compared to combinations that include VCTE plus Fib-4 or LB. The results highlight the importance of diagnostic accuracy, with costs being more influential on strategies including MRE. This finding suggests that, due to its point-of-care availability and more affordable cost, a VCTE based strategy may be the most attractive approach when considering potential population-based screening program as well as in the setting of specialist clinics. 
If the goal is to avoid liver biopsy, Fib-4+VCTE is a very cost-effective strategy among NITs. This strategy could be particularly important in the community setting or in resource-limited areas where Fib-4+VCTE can be used to screen for cirrhosis among patients with NAFLD. In the setting of higher prevalence of cirrhosis such as referral centers, either Fib-4 together with VCTE or MRE may be useful tools for detecting cirrhosis.

This analysis evaluated the cost-effectiveness of a comprehensive list of screening strategies for cirrhosis diagnosis in the context of NAFLD with varying prevalence of cirrhosis. Other strengths of this study include: (1) using liver biopsy as the reference standard for cirrhosis diagnosis and blood- and imaging-based tests, (2) simulated distribution of outcomes and its treatments considering real-world data, and (3) assessment of uncertainty including a wide range of cirrhosis prevalence, sensitivity and specificity values, as well as assuring the statistical confidence in comparative studies by running the microsimulation with a sufficiently large cohort multiple times for each strategy.

This analysis has limitations as well, including: (1) results are based on the U.S. costs and may not generalize to other healthcare systems; (2) MRI costs were used as a proxy for MRE; MRE is a relatively new diagnostic imaging technology without a CPT code for insurance reimbursement currently, although it is being used often in the United States for routine clinical care; (3) since the sensitivity and specificity of non-invasive tests in the context of a primary care setting are not available, this information was extrapolated from studies conducted at tertiary referral centers; (4) in order to minimize the complexity of our analysis, we simplified some aspects of our decision tree such as single time testing and binary cut-off values for VCTE and MRE and assuming that causes of death are limited to variceal bleeding and HCC during first five years of 
follow-up; and (5) finally, our cost-effectiveness is not a traditional one, i.e., cost per life-year save or cost per quality adjusted life year. We used cost per correct diagnosis as a proxy for costeffectiveness for several reasons. such that the risk of hard outcomes such as mortality due to hepatic and cardiovascular events is not common. Second, not well-described are the natural history of cirrhosis due to NAFLD and the effects of identifying and treating large esophageal varices and hepatocellular carcinoma in this setting.

In conclusion, this study suggests that Fib-4 followed by either VCTE, MRE or LB are costeffective strategies for identifying cirrhosis in populations where the prevalence of cirrhosis varies between $0.27 \%-4 \%$. Compared to the combination of FIB-4 and VCTE, the ICERs were higher for the combination of FIB-4 and MRE were lower than for the combination of FIB-4 and liver biopsy. If the goal is to avoid liver biopsy, the combination of Fib-4+VCTE with its lower costs and accessibility is likely the preferred strategy for the screening of cirrhosis in the setting of general or community-based populations. 


\section{REFERENCES}

1. Chalasani N, Younossi Z, Lavine JE, et al. The diagnosis and management of nonalcoholic fatty liver disease: Practice guidance from the American Association for the Study of Liver Diseases. Hepatology 2018;67:328-357.

2. Vilar-Gomez E, Calzadilla-Bertot L, Wai-Sun Wong V, et al. Fibrosis Severity as a Determinant of Cause-Specific Mortality in Patients With Advanced Nonalcoholic Fatty Liver Disease: A Multi-National Cohort Study. Gastroenterology 2018;155:443-457 e17.

3. Sterling RK, Lissen E, Clumeck N, et al. Development of a simple noninvasive index to predict significant fibrosis in patients with HIV/HCV coinfection. Hepatology 2006;43:1317-25.

4. Vuppalanchi R, Siddiqui MS, Van Natta ML, et al. Performance characteristics of vibrationcontrolled transient elastography for evaluation of nonalcoholic fatty liver disease. Hepatology 2018;67:134-144.

5. Loomba R, Cui J, Wolfson T, et al. Novel 3D Magnetic Resonance Elastography for the Noninvasive Diagnosis of Advanced Fibrosis in NAFLD: A Prospective Study. American Journal of Gastroenterology 2016;111:986-994.

6. Loomba R, Wolfson T, Ang B, et al. Magnetic resonance elastography predicts advanced fibrosis in patients with nonalcoholic fatty liver disease: a prospective study. Hepatology 2014;60:1920-8.

7. Sun WJ, Cui HL, Li N, et al. Comparison of FIB-4 index, NAFLD fibrosis score and BARD score for prediction of advanced fibrosis in adult patients with non-alcoholic fatty liver disease: A meta-analysis study. Hepatology Research 2016;46:862-870.

8. Imajo K, Kessoku T, Honda Y, et al. Magnetic Resonance Imaging More Accurately Classifies Steatosis and Fibrosis in Patients With Nonalcoholic Fatty Liver Disease Than Transient Elastography. Gastroenterology 2016;150:626-637.

9. Petta S, Vanni E, Bugianesi E, et al. The combination of liver stiffness measurement and NAFLD fibrosis score improves the noninvasive diagnostic accuracy for severe liver fibrosis in patients with nonalcoholic fatty liver disease. Liver Int 2015;35:1566-73.

10. Tapper EB, Sengupta N, Hunink MG, et al. Cost-Effective Evaluation of Nonalcoholic Fatty Liver Disease With NAFLD Fibrosis Score and Vibration Controlled Transient Elastography. Am J Gastroenterol 2015;110:1298-304. 
11. Williams CD, Stengel J, Asike MI, et al. Prevalence of nonalcoholic fatty liver disease and nonalcoholic steatohepatitis among a largely middle-aged population utilizing ultrasound and liver biopsy: a prospective study. Gastroenterology 2011;140:124-31.

12. Scaglione $\mathrm{S}$, Kliethermes $\mathrm{S}, \mathrm{Cao} \mathrm{G}$, et al. The Epidemiology of Cirrhosis in the United States: A Population-based Study. J Clin Gastroenterol 2015;49:690-6.

13. Castera L, Foucher J, Bernard PH, et al. Pitfalls of liver stiffness measurement: a 5-year prospective study of 13,369 examinations. Hepatology 2010;51:828-35.

14. Chen J, Yin M, Talwalkar JA, et al. Diagnostic Performance of MR Elastography and Vibration-controlled Transient Elastography in the Detection of Hepatic Fibrosis in Patients with Severe to Morbid Obesity. Radiology 2017;283:418-428.

15. Yin M, Glaser KJ, Talwalkar JA, et al. Hepatic MR Elastography: Clinical Performance in a Series of 1377 Consecutive Examinations. Radiology 2016;278:114-24.

16. Heimbach JK, Kulik LM, Finn RS, et al. AASLD guidelines for the treatment of hepatocellular carcinoma. Hepatology 2018;67:358-380.

17. Garcia-Tsao G, Abraldes JG, Berzigotti A, et al. Portal hypertensive bleeding in cirrhosis: Risk stratification, diagnosis, and management: 2016 practice guidance by the American Association for the study of liver diseases. Hepatology 2017;65:310-335.

18. Nakamura S, Konishi H, Kishino M, et al. Prevalence of esophagogastric varices in patients with non-alcoholic steatohepatitis. Hepatology Research 2008;38:572-579.

19. Stevenson M, Lloyd-Jones M, Morgan MY, et al. Non-invasive diagnostic assessment tools for the detection of liver fibrosis in patients with suspected alcohol-related liver disease: a systematic review and economic evaluation. Health Technol Assess 2012;16:1-174.

20. Petta S, Sebastiani G, Bugianesi E, et al. Non-invasive prediction of esophageal varices by stiffness and platelet in non-alcoholic fatty liver disease cirrhosis. J Hepatol 2018;69:878885.

21. Merli M, Nicolini G, Angeloni S, et al. Incidence and natural history of small esophageal varices in cirrhotic patients. J Hepatol 2003;38:266-72.

22. Gluud LL, Krag A. Banding ligation versus beta-blockers for primary prevention in oesophageal varices in adults. Cochrane Database Syst Rev 2012:CD004544.

23. Cheng JW, Zhu L, Gu MJ, et al. Meta analysis of propranolol effects on gastrointestinal hemorrhage in cirrhotic patients. World J Gastroenterol 2003;9:1836-9. 
24. Majumdar A, Campos S, Gurusamy K, et al. Defining the Minimum Acceptable Diagnostic Accuracy of Noninvasive Fibrosis Testing in Cirrhosis: A Decision Analytic Modeling Study. Hepatology 2020;71:627-642.

25. Imperiale TF, Chalasani N. A meta-analysis of endoscopic variceal ligation for primary prophylaxis of esophageal variceal bleeding. Hepatology 2001;33:802-7.

26. Bedossa P, Dargere D, Paradis V. Sampling variability of liver fibrosis in chronic hepatitis C. Hepatology 2003;38:1449-57.

27. Singh S, Muir AJ, Dieterich DT, et al. American Gastroenterological Association Institute Technical Review on the Role of Elastography in Chronic Liver Diseases. Gastroenterology 2017; 152:1544-1577.

28. Hsu C, Caussy C, Imajo K, et al. Magnetic Resonance vs Transient Elastography Analysis of Patients With Nonalcoholic Fatty Liver Disease: A Systematic Review and Pooled Analysis of Individual Participants. Clin Gastroenterol Hepatol 2019;17:630-637 e8. 
Table 1. Model parameters, base-case values and ranges.

\begin{tabular}{lccc}
\hline Parameter & Value & $\begin{array}{c}\text { Range } \\
\text { (minimun-maximun) }\end{array}$ & Reference \\
\hline
\end{tabular}

Prevalence of biopsy-confirmed cirrhosis

Population-based

$0.27 \%$

12

Community-based

$2 \%$

11

$34.7 \%$

18

$11.5 \% \quad 20$

Prevalence of large or high-risk varices

$34 \%$

21

3-year rate of varices progression

$4.4 \%$

Annual incidence of esophageal varices

2-year risk of bleeding with primary prophylaxis

$17 \%$

$14 \%-20 \%$

$31 \%$

$18 \%-60 \%$

23,25

5-year mortality due to variceal bleeding

$20 \%-80 \%$

17

$20 \%$

$2.5 \%$

$49.2 \%$

$46.4 \%-77.2 \%$

2

3 -year mortality due to hepatocellular carcinoma

$49.2 \%$

16

Liver biopsy *

$93 \%$

Sensitivity

$95 \%$

$89 \%-100 \%$

Specificity

$92 \%-100 \%$

Fib-4 index

Optimal high cutoff (>3.25)

Sensitivity

$38 \%$

$35 \%-41 \%$

Specificity

$97 \%$

95\%-100\%

Optimal low cutoff $(<1.30)$

Sensitivity

$84 \%$

$74 \%-85 \%$

Specificity

$69 \%$

$65 \%-71 \%$

Indeterminate results

$32 \%$

$12 \%-46 \%$

7

Magnetic resonance elastography

Optimal cutoff for cirrhosis (4.7)

Sensitivity

$80 \%$

$60 \%-97 \%$

Specificity

$86 \%$

$84 \%-93 \%$

Failure rates

$4 \%$

$4 \%-6 \%$

14,15 
Vibration controlled transient elastography

Optimal cutoff for cirrhosis (11.8)

Sensitivity

$80 \%$

$78 \%-95 \%$

Specificity

$81 \%$

$85 \%-89 \%$

Failure rates

$7.1 \%$

$3.5 \%-50 \%$

4,13

* Considering optimal liver tissue specimens $(\geq 2.5 \mathrm{~cm}$ in length and $\geq 10$ portal tracts)

Specificity and sensitivity for cirrhosis were calculated using pooled data from published studies. 
Table 2. Accuracy and cost-effectiveness of different diagnostic strategies. A microsimulation analysis based on 100,000 NAFLD patients considering a cirrhosis prevalence of $0.27 \%$.

\begin{tabular}{|c|c|c|c|c|c|c|c|c|c|c|}
\hline Strategy & $\begin{array}{c}\text { Number of } \\
\text { correctly } \\
\text { identified } \\
\text { out of } 270 \\
\text { people with } \\
\text { cirrhosis }\end{array}$ & $\begin{array}{c}\text { Number of } \\
\text { correctly } \\
\text { excluded out of } \\
99730 \text { people } \\
\text { without } \\
\text { cirrhosis }\end{array}$ & $\begin{array}{c}\text { Percentage } \\
\text { of people } \\
\text { correctly } \\
\text { classified }\end{array}$ & $\begin{array}{l}\text { Change in } \\
\text { percentage } \\
\text { of people } \\
\text { correctly } \\
\text { classified* }\end{array}$ & $\begin{array}{c}\text { Cost } \\
\text { per } \\
\text { person } \\
(\$)\end{array}$ & $\begin{array}{c}\text { Cost per } \\
\text { correct } \\
\text { diagnosis } \\
(\$)\end{array}$ & $\begin{array}{l}\text { Mortality } \\
\text { (bleeding) }\end{array}$ & $\begin{array}{c}\text { Mortality } \\
\text { (HCC) }\end{array}$ & $\begin{array}{c}\text { Total } \\
\text { mortality } \\
\text { cases }\end{array}$ & ICER \\
\hline No test & N/A & N/A & N/A & N/A & 10 & N/A & 25 & 5 & 46 & - \\
\hline Fib-4+VCTE & 116 & 89,145 & $89.3 \%$ & $32.2 \%$ & 401 & 450 & 21 & 5 & 42 & Least costly \\
\hline Fib-4+MRE & 120 & 92,272 & $92.4 \%$ & $35.4 \%$ & 491 & 531 & 22 & 5 & 42 & 2,864 \\
\hline VCTE+LB & 187 & 91,769 & $92.0 \%$ & $34.9 \%$ & 612 & 667 & 19 & 4 & 40 & 4,454 \\
\hline Fib-4+LB & 145 & 97,592 & $97.7 \%$ & $40.7 \%$ & 729 & 747 & 21 & 5 & 42 & Dominated \\
\hline MRE+LB & 193 & 95,071 & $95.3 \%$ & $38.2 \%$ & 888 & 932 & 19 & 4 & 40 & Dominated \\
\hline VCTE & 201 & 75,046 & $75.2 \%$ & $18.2 \%$ & 900 & 1,197 & 18 & 4 & 39 & Dominated \\
\hline Fib-4 & 70 & 56,966 & $57.0 \%$ & Reference & 908 & 1,592 & 21 & 5 & 41 & Dominated \\
\hline MRE & 207 & 82,337 & $82.5 \%$ & $25.5 \%$ & 1,109 & 1,344 & 19 & 4 & 39 & Dominated \\
\hline LB & 251 & 94,744 & $95.0 \%$ & $38.0 \%$ & 1,663 & 1,751 & 17 & 4 & 37 & Dominated \\
\hline
\end{tabular}

Abbreviations: VCTE, vibration controlled transient elastography; LB, liver biopsy; MRE, Magnetic resonance elastography; HCC, hepatocellular carcinoma; ICER, incremental cost effectiveness ratio.

Total mortality includes mortality cases from variceal bleeding, HCC and other than bleeding or HCC.

Strategies are listed in order of increasing costs.

* It represents difference between tests and the reference strategy (Fib-4). 
Table 3. Accuracy and cost-effectiveness of different diagnostic strategies. A microsimulation analysis based on 100,000 NAFLD patients considering a cirrhosis prevalence of $2 \%$.

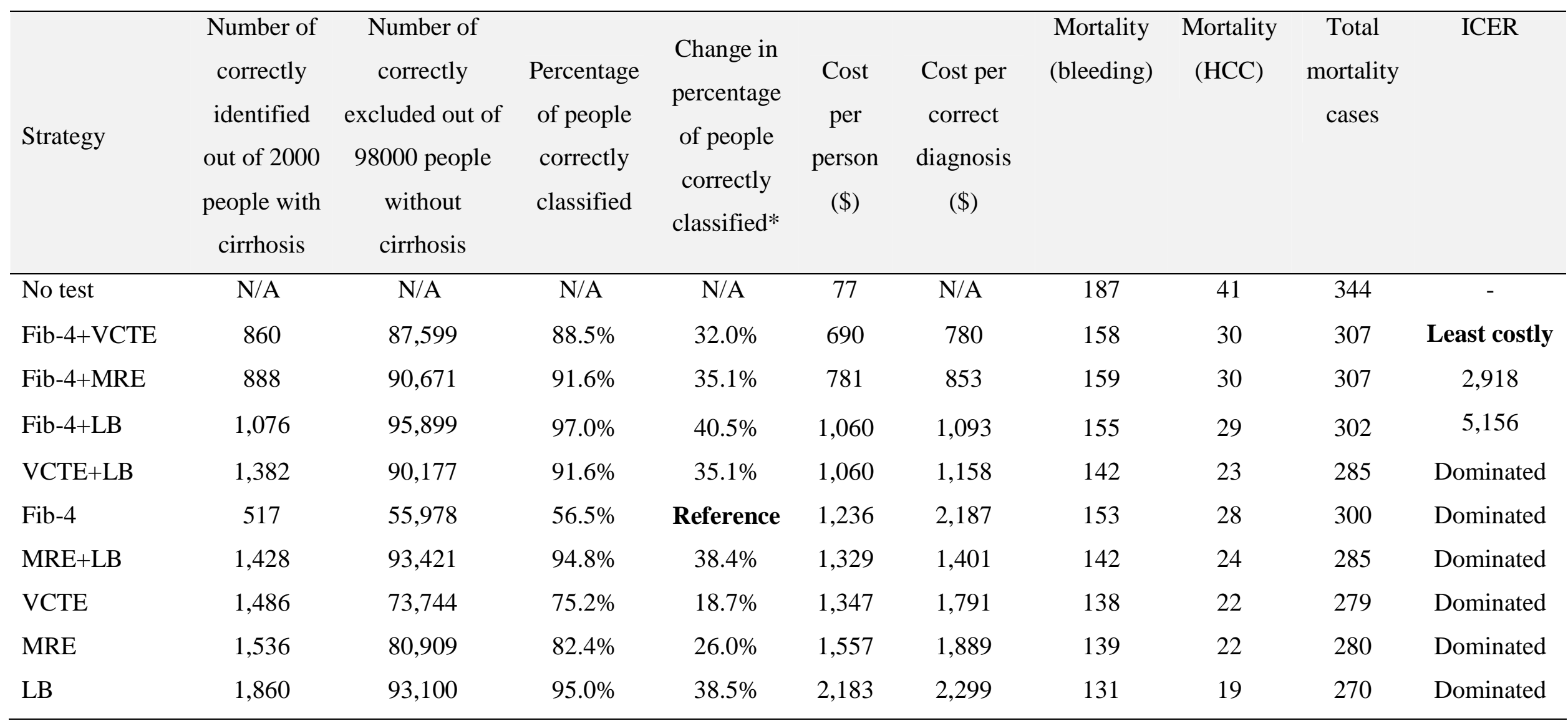

Abbreviations: VCTE, vibration controlled transient elastography; LB, liver biopsy; MRE, Magnetic resonance elastography; HCC, hepatocellular carcinoma; ICER, incremental cost effectiveness ratio.

Total mortality includes mortality cases from variceal bleeding, HCC and other than bleeding or HCC.

Strategies are listed in order of increasing costs.

* It represents difference between tests and the reference strategy (Fib-4). 
Table 4. Accuracy and cost-effectiveness of different diagnostic strategies. A microsimulation analysis based on 100,000 NAFLD patients considering a cirrhosis prevalence of $4 \%$.

\begin{tabular}{|c|c|c|c|c|c|c|c|c|c|c|}
\hline Strategy & $\begin{array}{c}\text { Number of } \\
\text { correctly } \\
\text { identified } \\
\text { out of } 4000 \\
\text { people with } \\
\text { cirrhosis }\end{array}$ & $\begin{array}{c}\text { Number of } \\
\text { correctly } \\
\text { excluded out of } \\
96000 \text { people } \\
\text { without } \\
\text { cirrhosis }\end{array}$ & $\begin{array}{c}\text { Percentage } \\
\text { of people } \\
\text { correctly } \\
\text { classified }\end{array}$ & $\begin{array}{l}\text { Change in } \\
\text { percentage } \\
\text { of people } \\
\text { correctly } \\
\text { classified* }\end{array}$ & $\begin{array}{c}\text { Cost } \\
\text { per } \\
\text { person } \\
(\$)\end{array}$ & $\begin{array}{c}\text { Cost per } \\
\text { correct } \\
\text { diagnosis } \\
(\$)\end{array}$ & $\begin{array}{l}\text { Mortality } \\
\text { (bleeding) }\end{array}$ & $\begin{array}{c}\text { Mortality } \\
\text { (HCC) }\end{array}$ & $\begin{array}{c}\text { Total } \\
\text { mortality } \\
\text { cases }\end{array}$ & ICER \\
\hline No test & N/A & N/A & N/A & N/A & 157 & N/A & 373 & 81 & 686 & - \\
\hline Fib-4+VCTE & 1872 & 85659 & $87.5 \%$ & $31.7 \%$ & 1024 & 1170 & 316 & 60 & 613 & Least costly \\
\hline Fib-4+MRE & 1863 & 88735 & $90.6 \%$ & $34.7 \%$ & 1114 & 1230 & 318 & 60 & 613 & 2,921 \\
\hline Fib-4+LB & 2152 & 93942 & $96.1 \%$ & $40.2 \%$ & 1441 & 1500 & 310 & 57 & 603 & 5,956 \\
\hline VCTE+LB & 3029 & 88073 & $91.1 \%$ & $35.2 \%$ & 1579 & 1733 & 283 & 47 & 568 & Dominated \\
\hline Fib-4 & 2224 & 53645 & $55.9 \%$ & Reference & 1616 & 2892 & 306 & 56 & 599 & Dominated \\
\hline $\mathrm{MRE}+\mathrm{LB}$ & 3006 & 91366 & $94.4 \%$ & $38.5 \%$ & 1840 & 1950 & 284 & 47 & 569 & Dominated \\
\hline VCTE & 3237 & 71975 & $75.2 \%$ & $19.3 \%$ & 1861 & 2474 & 276 & 44 & 559 & Dominated \\
\hline MRE & 3221 & 79109 & $82.3 \%$ & $26.5 \%$ & 2077 & 2523 & 277 & 44 & 559 & Dominated \\
\hline LB & 3720 & 91200 & $94.9 \%$ & $39.1 \%$ & 2777 & 2925 & 262 & 39 & 540 & Dominated \\
\hline
\end{tabular}

Abbreviations: VCTE, vibration controlled transient elastography; LB, liver biopsy; MRE, Magnetic resonance elastography; HCC, hepatocellular carcinoma; ICER, incremental cost effectiveness ratio.

Total mortality includes mortality cases from variceal bleeding, HCC and other than bleeding or HCC.

Strategies are listed in order of increasing costs.

* It represents difference between tests and the reference strategy (Fib-4). 


\title{
FIGURE LEGENDS
}

Fig. 1. Model structure.
(A) Decision analytic tree using single tests.
(B) Decision analytic tree using sequential tests.
(C) Microsimulation of patients' assessment after receiving a diagnosis of cirrhosis.

\begin{abstract}
Abbreviations: VCTE, vibration controlled transient elastography; MRE, magnetic resonance elastography; $\mathbf{C}$, cirrhosis; NC, no cirrhosis; CD, correctly diagnosed; MD, misdiagnosed; CR, correctly ruled out; UD, undiagnosed; HCC, hepatocellular carcinoma; EV, esophageal varices; EVL, endoscopic variceal ligation; OLT, orthotopic liver transplantation; NSBB, nonselective beta-blockers; EGD, esophagogastroduodenoscopy.
\end{abstract}

Fig. 2. Incremental cost-effectiveness or cost-mortality "Frontier" of 9 diagnostic strategies.
(A) Cost per person vs accuracy considering a cirrhosis prevalence of $0.27 \%$.
(B) Cost per person vs mortality considering a cirrhosis prevalence of $0.27 \%$.
(C) Cost per person vs accuracy considering a cirrhosis prevalence of $2 \%$.
(D) Cost per person vs mortality considering a cirrhosis prevalence of $2 \%$.
(E) Cost per person vs accuracy considering a cirrhosis prevalence of $4 \%$.
(F) Cost per person vs mortality considering a cirrhosis prevalence of $4 \%$.

The points on the line are the strategies on the frontier and considered dominating strategies, and points below the line are the less cost-effective strategies.

\footnotetext{
Abbreviations: VCTE, vibration controlled transient elastography; LB, liver biopsy; MRE, magnetic resonance elastography.
} 
Figure $1 \mathrm{~A}$

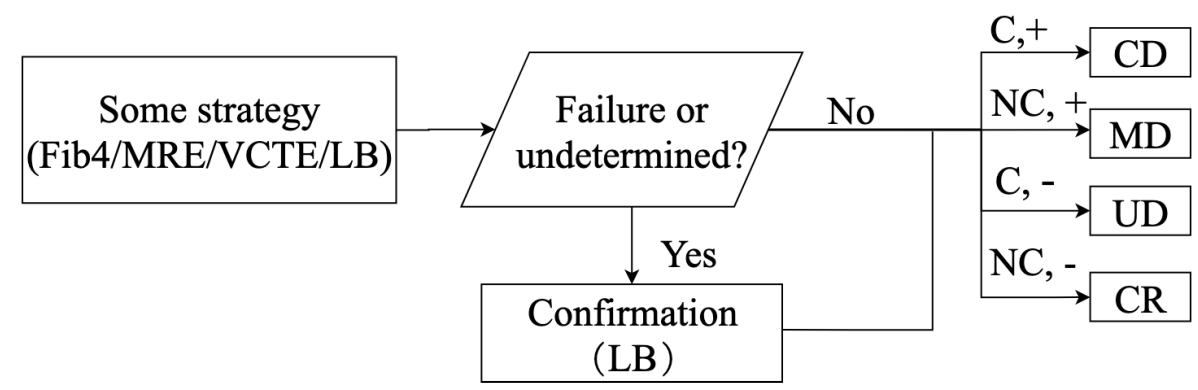


Figure 1B

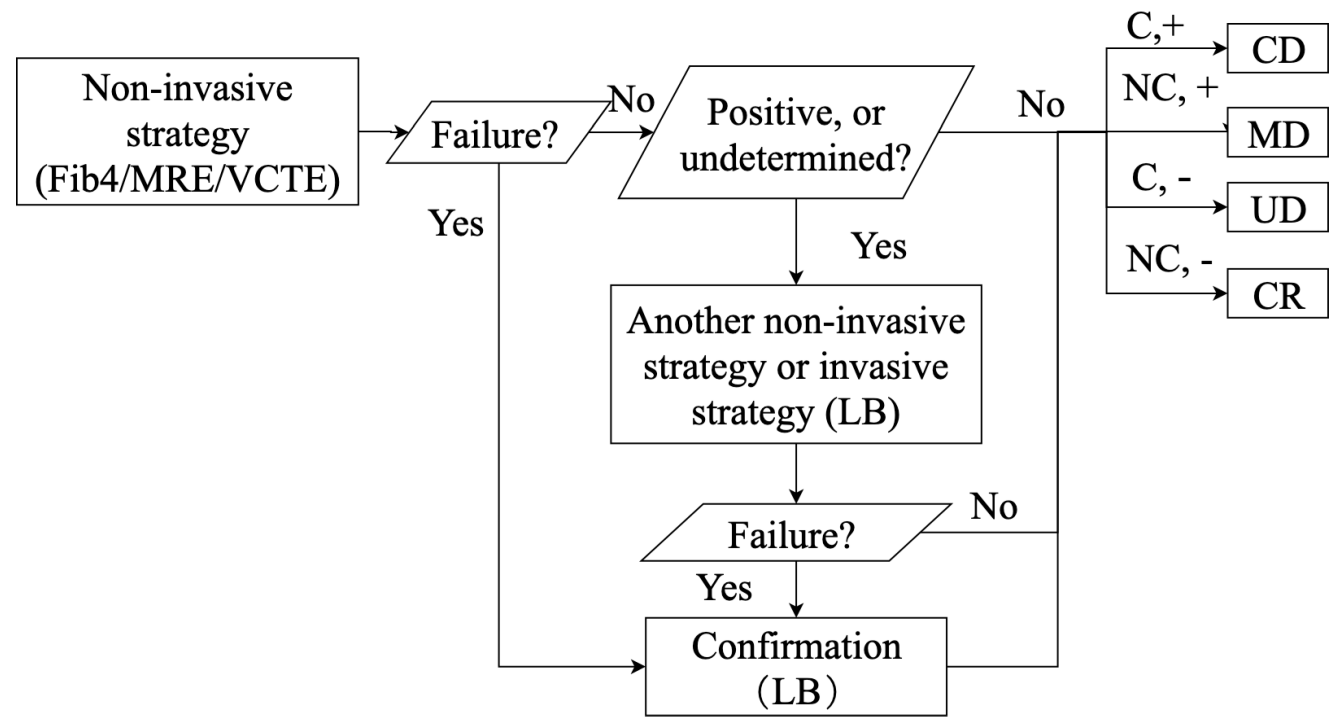




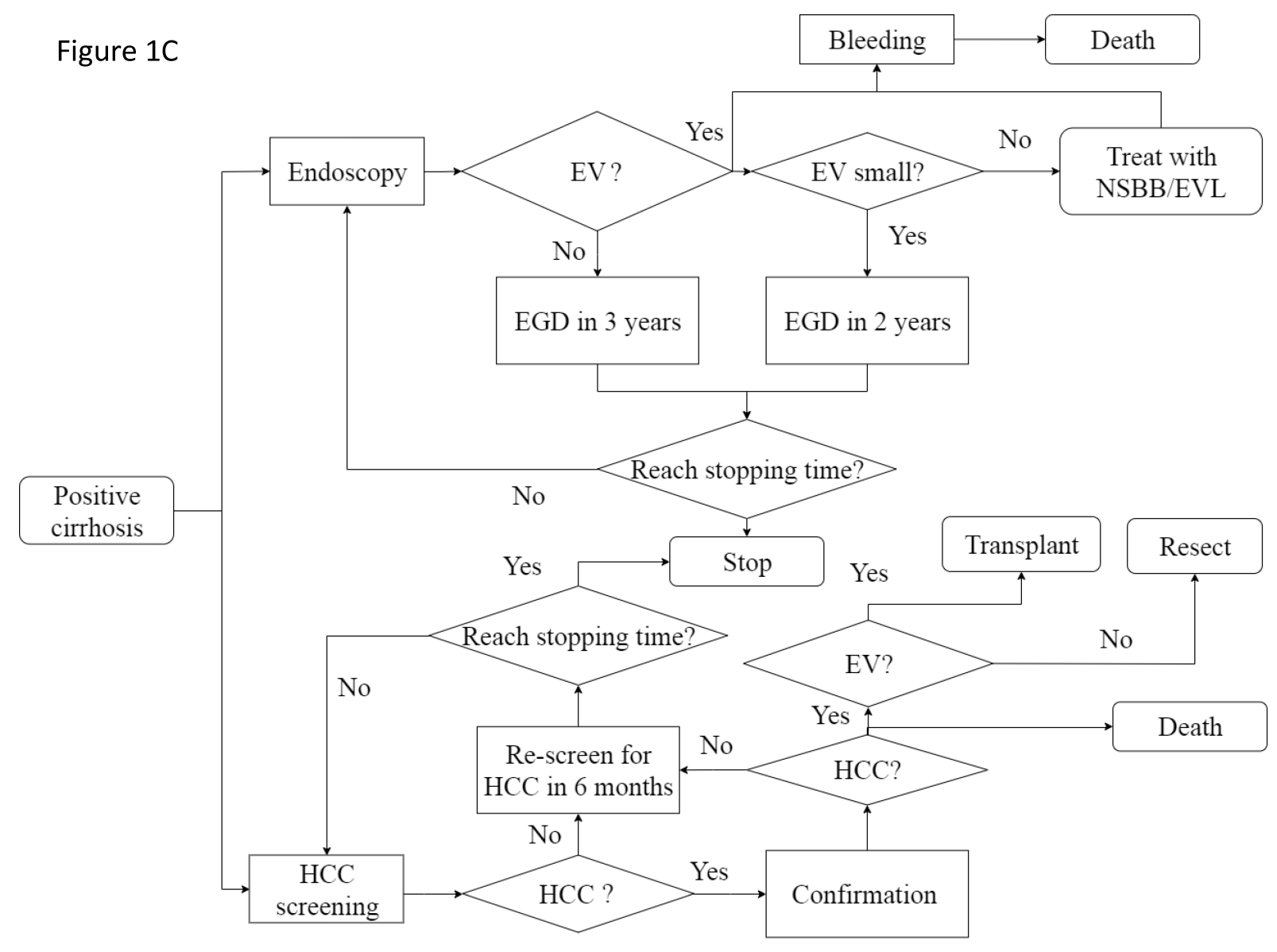


Figure 2A

\section{Frontier (Prevalence .27\%)}

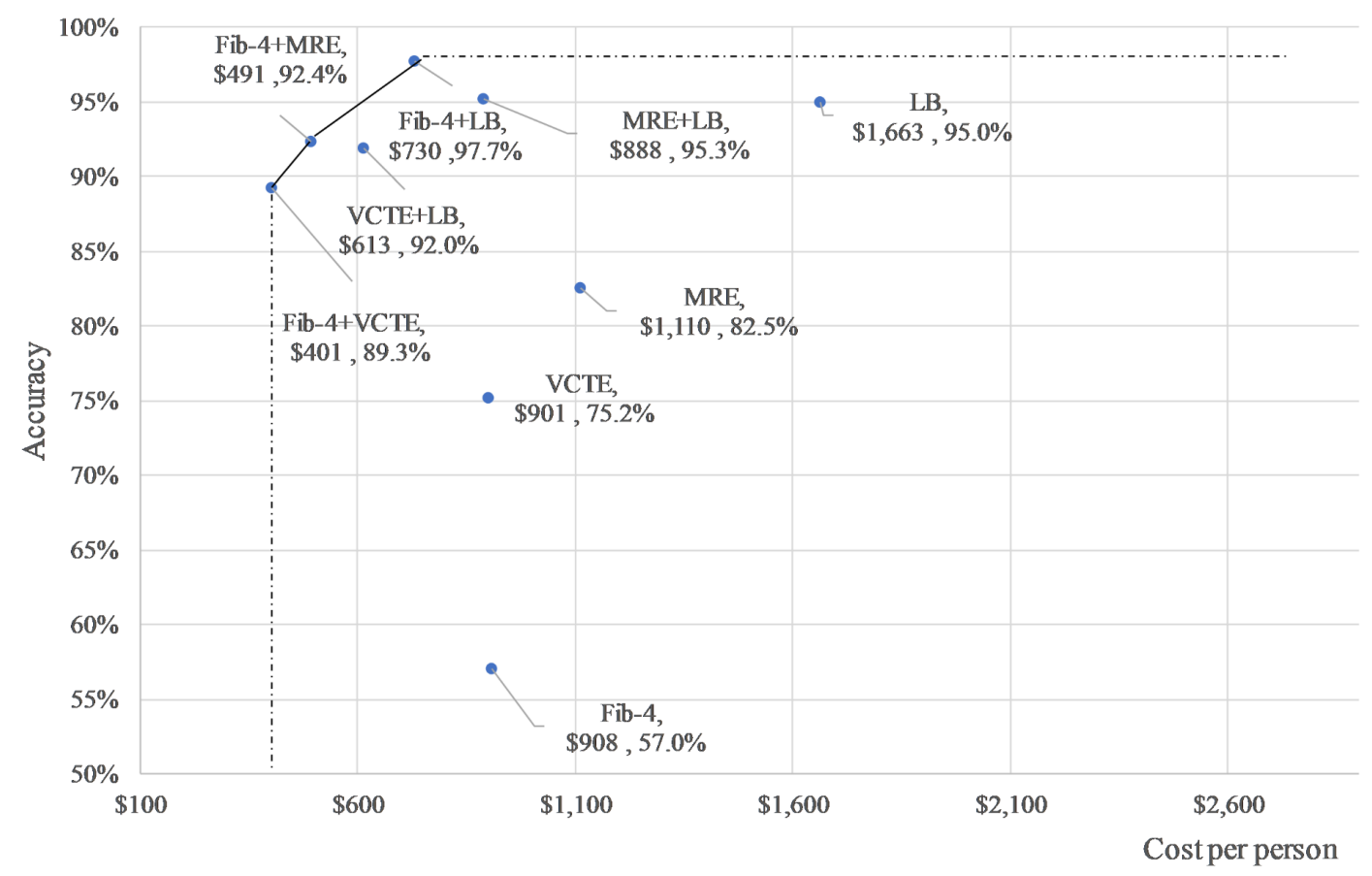


Figure 2B

Frontier (Prevalence .27\%)

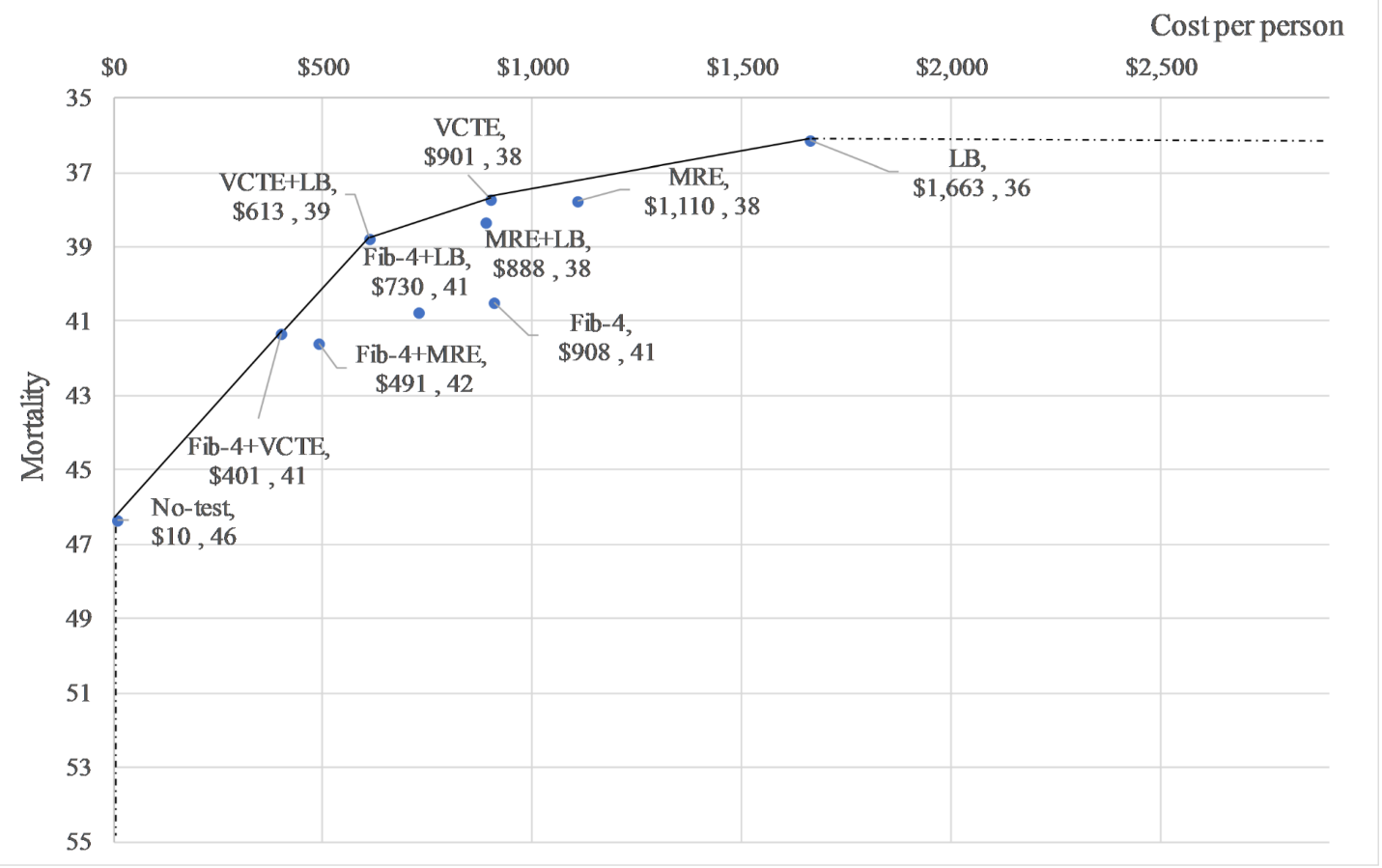


Figure 2C

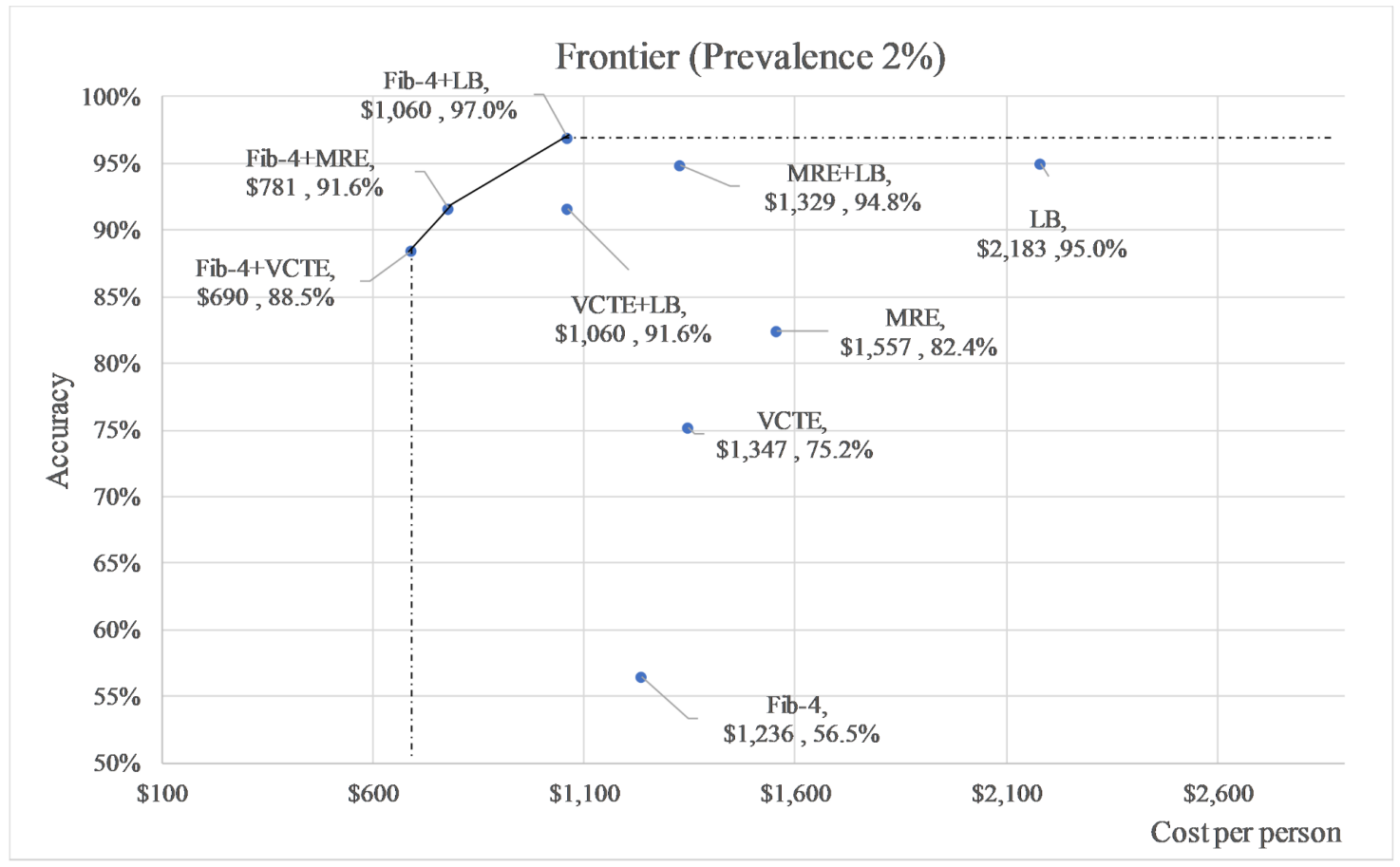


Figure 2D

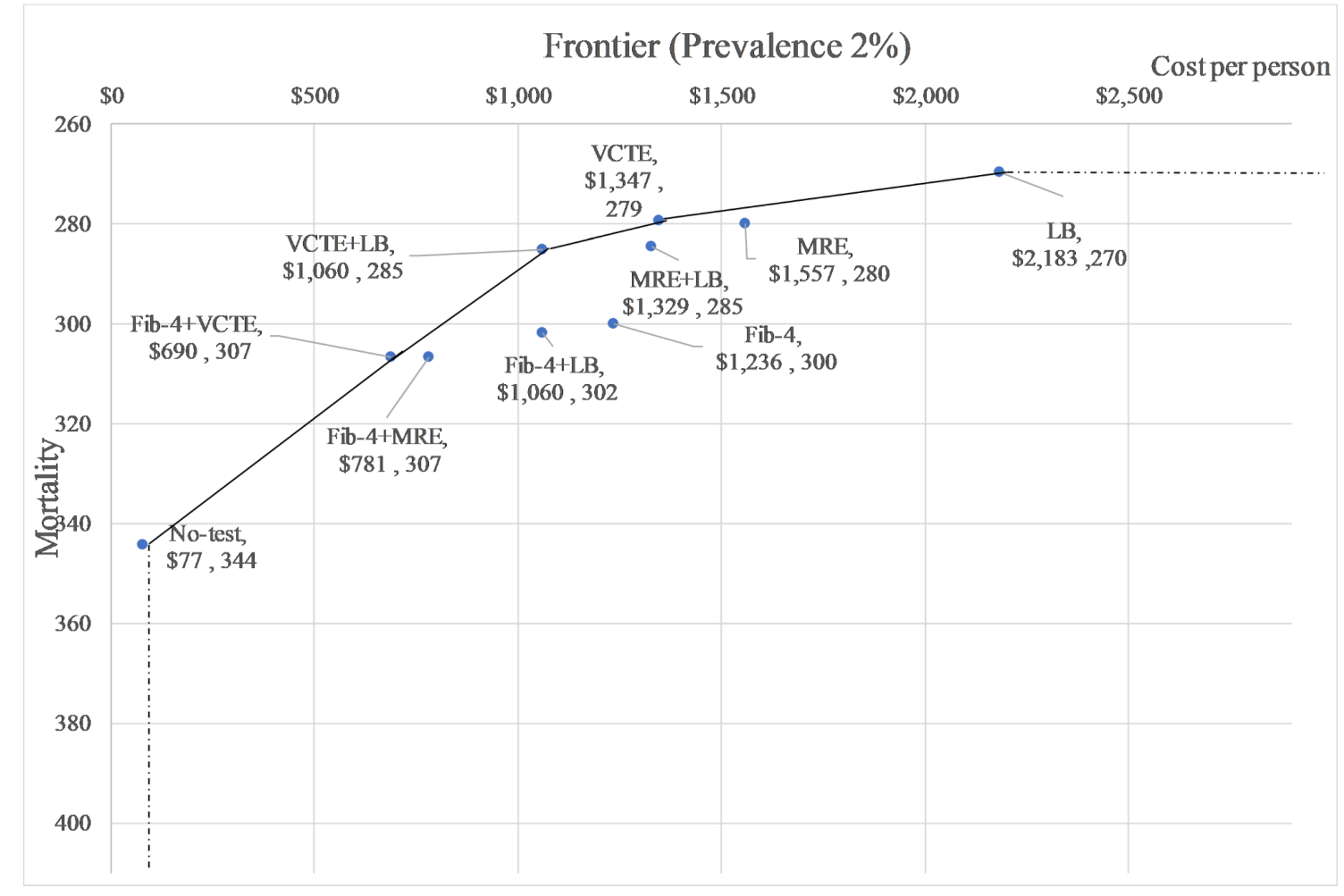


Figure 2E

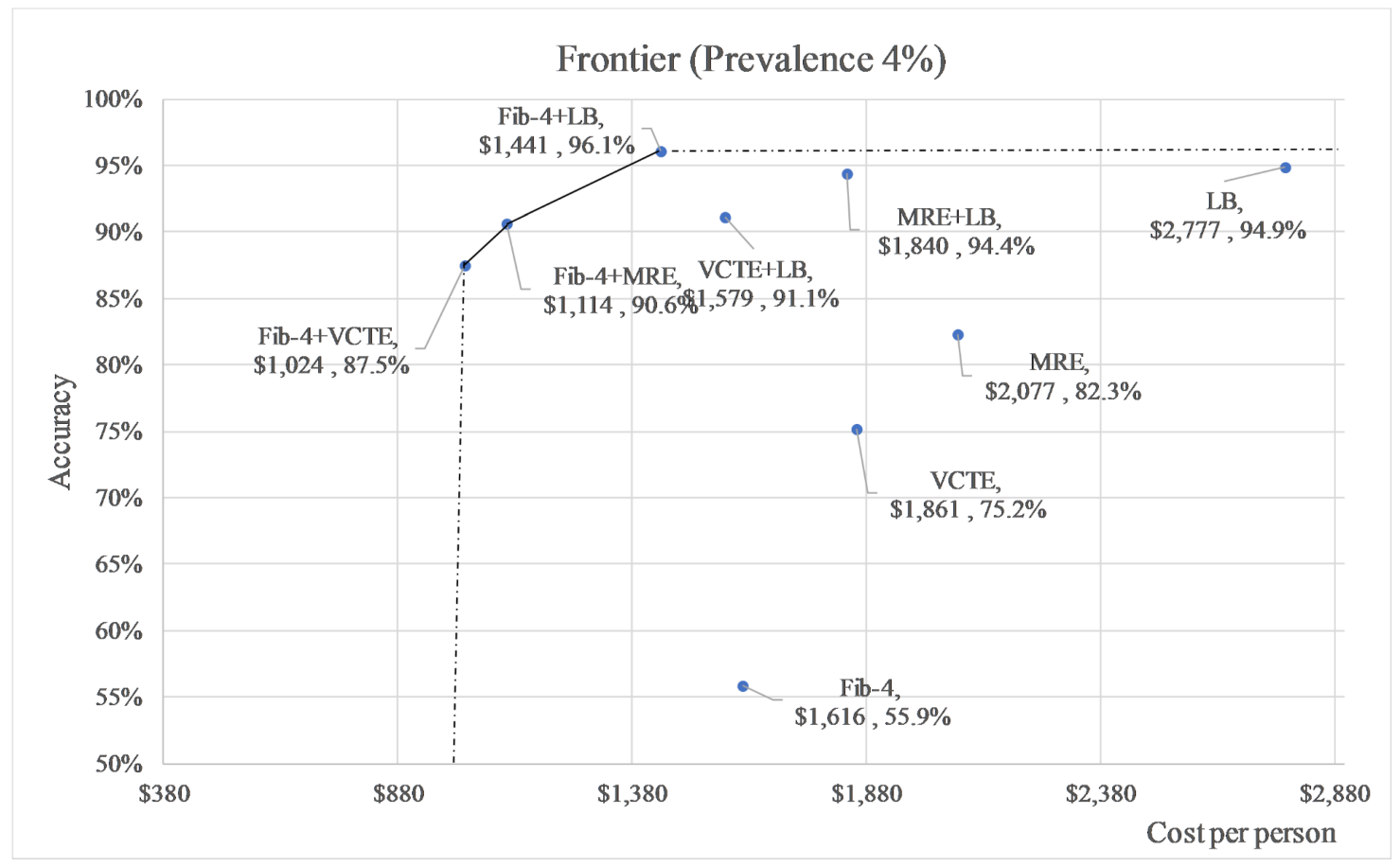


Figure 2F

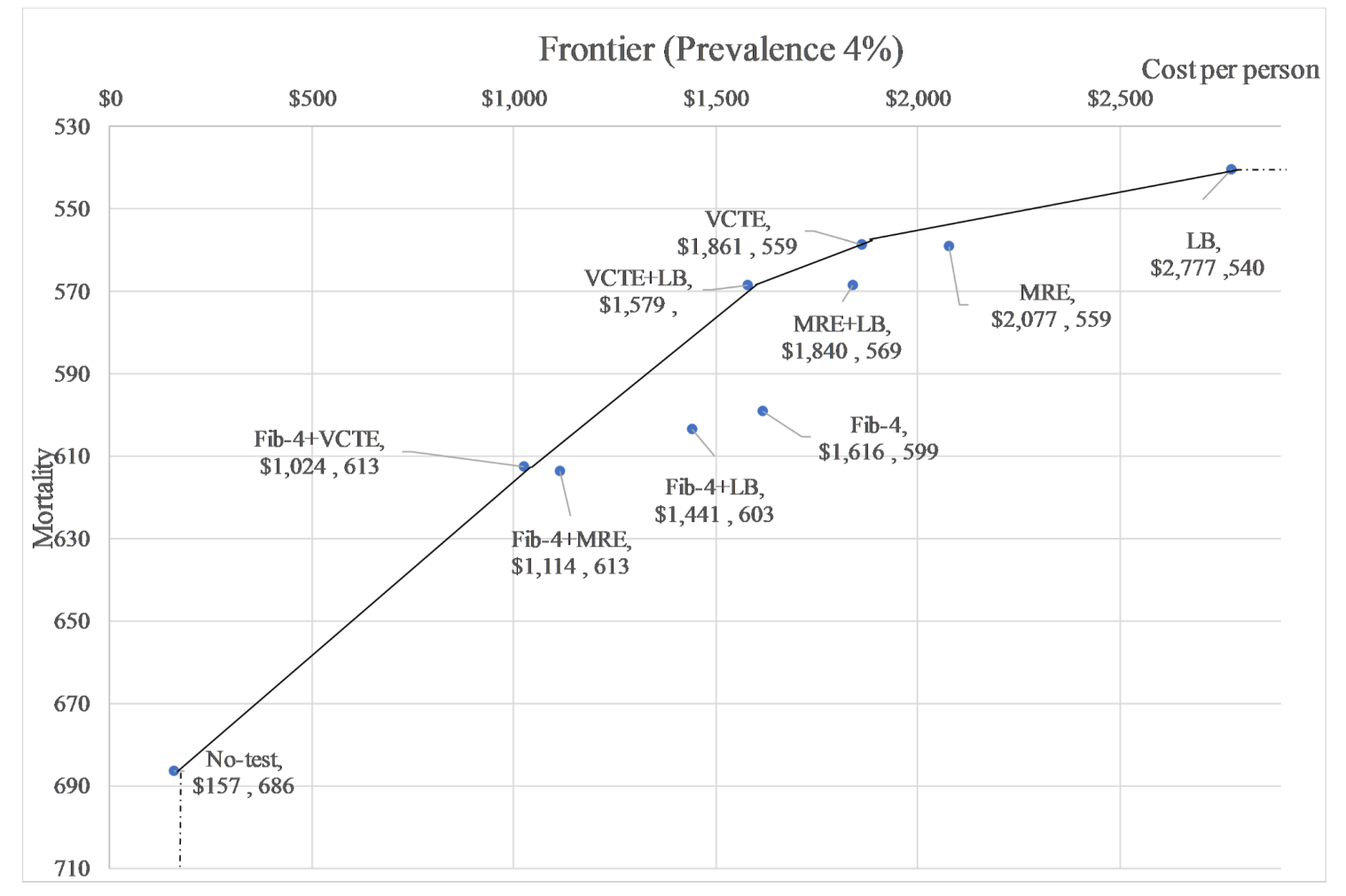

